\title{
A Class of Scale Mixtured Normal Distribution
}

\section{Rezaul Karim Md*}

Department of Statistics, Jahangirnagar University, Bangladesh

\begin{abstract}
A class of scale mixtured normal distribution with lifetime probability distributions has been proposed in this article. Different moments, characteristic function and shape characteristics of these proposed probability distributions have also been provided. The scale mixture of normal distribution is extensively used in Biostatistical field. In this article, some new lifetime probability distributions have been proposed which is the scale mixture of normal distribution. Different properties such as moments, characteristic function, shape characteristics of these probability distributions have also been mentioned.
\end{abstract}

Keyword: Scale mixture distribution; Inverted probability distribution; Moments; Skewness; Kurtosis

\section{Introduction}

A mixture distribution is a convex function of a set of probability distributions [1]. At first, Pearson considered a mixture distribution of two normal distributions in 1894 and he studied the estimation of the parameters of his proposed distribution [2]. After around half century, some characteristics of mixture distributions were mentioned by Robins (1948). Some of researchers like Rider, Blichke, Karim [1,3-5] studied the various mixture of distributions. However, they studied only for location mixture of the distributions though the scale mixture of the distributions have widely used in biostatistical field. The location testing for Normal scale mixture distributions obtains in some fastidious types of situations where the student's t-test is inapplicable. Specifically, these cases permit tests of location to be prepared where the assumption that sample observations obtain form populations having a Gaussian distribution can be replaced by assumption that they arise from a Normal scale mixture distribution. A few researchers [3,6-8] mentioned the definition of scale mixture of normal distributions with its applications. There are many applications of scale mixture of normal distribution with lifetime probability distribution in biostatistical field whereas; only the scale mixture of normal distribution is familiar for us. The scale mixture of normal distribution with lifetime distributions are not only used in biological field but also used in fisheries, agricultural, psychological, paleontological field. It is also widely used in electrophoresis, and communication theories. In this paper, the definition of the scale mixture of normal distribution with a set of lifetime probability distributions is defined initially and then scale mixture of normal distribution with inverted Chi-square, Rayleigh, Exponential, Gamma and Erlang distributions are studied. The different properties of these distributions are also presented.

\section{Preliminaries}

A mixture distribution is the probability distribution of a random variable whose values can be taken in a simple way from an underlying set of other random variables, with a certain probability of selection being associated with each. Suppose the random variable $\mathrm{X}$ has a probability density function $(P d f) f(x \mid \theta)$. The sampling distribution of a statistic of the parameter $\theta$ is $\tau(\theta)$ where $\Theta$ is the parameter space of $\theta$. And then, a continuous mixture of densities $f(x \mid \theta)$ with weight functions $\tau(\theta)$ is expressed by following:

$$
m(x)=\int_{\Theta} f(x \mid \theta) \tau(\theta) d \theta
$$

\section{Main results}

Here, the definition of scale mixture of normal distribution with well known lifetime distributions such as inverted chi-square, gamma, exponential, Rayleigh distributions are mentioned. The important characteristics of these distributions such as characteristic functions, moments, and shape characteristics are also mentioned. The main results of the paper are presented in the form of definitions and theorems.

\section{Definition 3.1}

A continuous random variable $X$ is said to have a scale mixture of normal distribution if its probability density function is given by

$$
f\left(x ; \mu, \sigma^{2}\right)=\int_{0}^{\infty} \frac{1}{\tau \sigma \sqrt{2 \pi}} e^{-\frac{1}{2}\left(\frac{x-\mu}{\sigma}\right)^{2}} h(\tau) d \tau ; \quad-\infty<x<\infty
$$

Where, the parameters $\mu$ and $\sigma^{2}$ satisfy $-\infty<\mu<\infty$ and $\sigma^{2}>0$. Here $h(\tau)$ is a probability function of a random variable $\tau$. The variable $X$ whose density function (3.1) is called a scale mixture normal variate which can be written as $\mathrm{X} \mid \tau \sim N\left(\mu, \tau^{2} \sigma^{2}\right)$

\section{Definition 3.2}

If a random variable $X$ has a probability density function

$$
f(x)=\frac{1}{\sqrt{n} \sigma \beta\left(\frac{n}{2}, \frac{1}{2}\right)} \frac{1}{\left[\left(\frac{x-\mu}{\sigma}\right)^{2} / n\right]^{\frac{n+1}{2}}} ;-\infty<x<\infty
$$

then it said to be scale mixture of normal distribution with inverted chi-square distribution with location parameter $\mu$, scale parameter $\sigma^{2}$ and shape parameter $\beta$. Various moments and shape characteristic of this probability distribution are presented by following theorem.

\section{Theorem 3.1}

The moments and shape characteristics of scale mixture of normal

*Corresponding author: Rezaul Karim Md, Department of Statistics, Jahangirnagar University, Bangladesh, Tel: 880-2-7791045-51; E-mail: rezaul@juniv.edu

Received February 27, 2016; Accepted March 01, 2016; Published March 08 2016

Citation: Rezaul Karim Md (2016) A Class of Scale Mixtured Normal Distribution J Biom Biostat 7: 285. doi:10.4172/2155-6180.1000285

Copyright: @ 2016 Rezaul Karim Md. This is an open-access article distributed under the terms of the Creative Commons Attribution License, which permits unrestricted use, distribution, and reproduction in any medium, provided the original author and source are credited. 
distribution with inverted chi-square distribution are given by

$$
\mu_{2 r}=\frac{n^{r} \sigma^{2 r} \sqrt{\frac{n}{2}-r \sqrt{r+\frac{1}{2}}}}{\sqrt{\frac{n}{2} \sqrt{\frac{1}{2}}}} ; \mu_{2 r+1}=0
$$

Mean $=0$, Variance $=\frac{n}{n-2} \sigma^{2}$

Skewness: $\beta_{1}=0$, Kurtosis: $\beta_{2}=\frac{3(n-2)}{n-4}$.

\section{Definition 3.3}

A random variable $X$ said to be scale mixture of normal distribution with inverted Gamma distribution if its probability density function is following

$$
f(x)=\frac{\sqrt{n \beta}}{\sigma \sqrt{2} B\left(n, \frac{1}{2}\right)} \frac{1}{\left[\left(\frac{x-\mu}{\sigma}\right)^{2} / 2 n \beta+1\right]^{n+\frac{1}{2}}} ;-\infty<x<\infty
$$

Where, $\mu, \sigma^{2}$ and $\beta$ represent location, scale and shape parameters respectively.

\section{Theorem 3.2}

The main features such as $r^{\text {th }}$ central moment, Skewness, kurtosis, etc. of scale mixture of normal distribution with inverted Gamma distribution are following:

$$
\begin{aligned}
& \mu_{2 r}=\frac{(2 r) !}{2^{r} r !} \sigma^{2 r}(n \beta)^{r} \frac{\sqrt{n-r}}{\sqrt{n}} ; \mu_{2 r+1}=0 ; \\
& \text { Mean= 0, variance }=\frac{n \beta}{n-1} \sigma^{2} \\
& \text { Skewness }=0, \text { Kurtosis }=\frac{3(n-1)}{(n-2)} .
\end{aligned}
$$

\section{Definition 3.4}

The probability density function of a random variable $X$ is said to have a scale mixture of normal distribution with inverted Rayleigh distribution if its probability density function is defined by

$$
f(x)=\frac{1}{\sigma \theta^{2}\left[\left(\frac{x-\mu}{\sigma}\right)^{2}+\frac{2}{\theta^{2}}\right]^{3 / 2}} ;-\infty<x<\infty
$$

Where $\theta$ is the parameter which satisfies $\theta>0$.

\section{Theorem 3.3}

The main properties of scale mixture normal distribution with inverted Rayleigh distribution are given by following:

$$
\mu_{2 r}=\frac{(2 r) !}{2^{r} r !} \sigma^{2 r} \frac{\overline{1-r}}{\theta^{2 r}} ; \mu_{2 r+1}=0 ;
$$

Mean $=0$ and variance $=\frac{\sigma^{2}}{\theta^{2}}$.

\section{Definition 3.5}

The probability density function of scale mixture normal distribution with inverted exponential distribution is as follows

$$
f(x)=\frac{\theta}{\sigma\left[\left(\frac{x-\mu}{\sigma}\right)^{2}+2 \theta\right]^{3 / 2}},-\infty<x<\infty
$$

Where $\mu, \sigma^{2}$ and $\beta$ represent location, scale and shape parameters respectively.

\section{Theorem 3.4}

The main characteristics of scale mixture normal distribution with inverted exponential distribution are

$$
\left.\mu_{2 r}=\frac{(2 r) !}{2^{r} r !} \sigma^{2 r} \theta^{r}\right) \overline{1-r} ; \mu_{2 r+1}=0 ;
$$

Mean $=0$ and variance $=\sigma^{2} \theta$.

\section{Definition 3.6}

Let $X$ be a continuous random variable. The probability density function of $X$ is said to be a scale mixture normal distribution with inverted Erlang distribution if its follows:

$$
f(x)=\frac{\sqrt{\lambda \theta}}{\sigma \sqrt{2} B\left(n, \frac{1}{2}\right)} \frac{1}{\left[\left(\frac{x-\mu}{\sigma}\right)^{2} / 2 \lambda \theta+1\right]^{\theta+\frac{1}{2}}} ;-\infty<x<\infty
$$

\section{Theorem 3.5}

The different characteristics such as moments, skewnbess and Kurtosis of the scale mixture normal distribution with inverted Erlang distribution are following:

$$
\begin{aligned}
& \qquad \mu_{2 r, m}=\frac{(2 r) !}{2^{r} r !} \sigma^{2 r}(\lambda \theta)^{r} \frac{\sqrt{\theta-r}}{\sqrt{\lambda}} ; \mu_{2 r+1}=0 ; \text { Mean }=0, \text { variance }= \\
& \frac{\lambda \theta}{\theta-1} \sigma^{2} \\
& \text { Skewness }=0 \text {, Kurtosis }=\frac{3(\theta-1)}{(\theta-2)} . \\
& \text { Concluding Remarks }
\end{aligned}
$$

\section{Concluding Remarks}

This article has been proposed a class of scale mixtured of normal distribution with different lifetime probability distribution such as inverted Rayleigh distribution, Erlang distribution and explained some basic properties of these proposed probability distributions with applications in various fields of applied science and business. I believe, this paper will help and encourage young researchers to stimulate further research.

\section{References}

1. Karim MR, Hossain MP, Begum S, Hossain MF (2011) Rayleigh Mixture Distribution. Journal of Applied Mathematics.

2. Pearson K (1894) Contribution to the mathematical theory of evolution Philosophical Transaction Royal Society. 185: 71-110.

3. Andrews DF, Mallows CL (1974) Scale Mixtures of Normal Distribution. Journa of the Royal Statistical Society 36: 99-102.

4. Rider PR (1961) The Method of Moments Applied To A Mixture of Two Exponential Distributions. The Annals of Mathematical Statistics 32: 143-147.

5. Blichke WR (1965) Mixtures of Discrete Distribution, Classical and Contagious Discrete Distributions. Calcutta Statistical Publishing Society Ed GP Patil 351- 372.

6. Choy STB, Jennifer SKC (2008) Scale Mixtures Distributions in Statistical Modelling. Australian \& NewZealand Journal of Statistics 50: 135-146.

7. West M (1987) On Scale Mixtures of Normal Distributions. Biometricka 74 646-648.

8. Monahan JF, Stefanski LA (1989) Normal scale mixture approximations to the logistic distribution with applications. Institute of Statistics Mineograph Series. 